\title{
A Space Vector PWM Scheme for Multifrequency Output Voltage Generation With Multiphase Voltage-Source Inverters
}

\author{
Drazen Dujic, Student Member, IEEE, Gabriele Grandi, Member, IEEE, \\ Martin Jones, Member, IEEE, and Emil Levi, Senior Member, IEEE
}

\begin{abstract}
Multiphase variable-speed drives, supplied from two-level voltage-source inverters (VSIs), are nowadays considered for various industrial applications. Depending on the drive structure and/or the motor design, the VSI is required to generate either sinusoidal voltages or voltages that contain a certain number of sinusoidal components ("multifrequency output voltages"). The existing space vector pulsewidth-modulation (SVPWM) schemes are based on selection of $(n-1)$ active space vectors (for odd phase numbers) within a switching period and they yield either sinusoidal voltage or sinusoidal fundamental voltage in combination with a limited amount of other harmonic terms. This paper develops a SVPWM scheme, which enables multifrequency output voltage generation with arbitrary values of various sinusoidal components in the output voltage. The method is based on initial selection of $(n-1)^{2} / 2$ active space vectors within a switching period, instead of the common $(n-1)$ active vectors. By properly arranging the sequence of the vector application, it is possible to provide an automatic postreduction of the number of applied active vectors to $(n-1)$, thus maintaining the same switching frequency as with the existing schemes while simultaneously avoiding the limiting on the generated sinusoidal output voltage components. Theoretical considerations are detailed using a five-phase VSI. The experimental verification is provided using a five-phase two-motor series-connected induction motor drive, supplied from a custom-designed five-phase DSPcontrolled VSI.
\end{abstract}

Index Terms-Multiphase variable-speed drives, multiphase voltage-source inverters (VSIs), space vector pulsewidth modulation (SVPWM).

\section{INTRODUCTION}

$\mathbf{R}$ ECENT developments in the area of multiphase variablespeed drives, initiated predominantly by potential applications in electric ship propulsion, more-electric aircraft, locomotive traction, electric and hybrid-electric vehicles, and other high power industries [1], have led to a corresponding development of pulsewidth modulation (PWM) schemes for

Manuscript received February 27, 2007; revised July 3, 2007. This work was supported in part by the Engineering and Physical Sciences Research Council under Grant EP/C007395, in part by Semikron, U.K., in part by MOOG, Italy, and in part by Verteco, Finland.

D. Dujic, M. Jones, and E. Levi are with the School of Engineering, Liverpool John Moores University, Liverpool, L3 3AF, U.K. (e-mail: e.levi@ ljmu.ac.uk).

G. Grandi is with the Department of Electrical Engineering, University of Bologna, 40136 Bologna, Italy (e-mail: gabriele.grandi@mail.ing.unibo.it).

Color versions of one or more of the figures in this paper are available online at http://ieeexplore.ieee.org.

Digital Object Identifier 10.1109/TIE.2008.918468 multiphase inverters used in these drives. By and large, the emphasis has been placed on space vector PWM (SVPWM) methods. Since an $n$-phase system corresponds to an $(n-1)$ dimensional space ( $n$ is further on assumed to be an odd prime number and the star-connected load is balanced with isolated neutral point), the customary approach toward designing an SVPWM scheme consists in decomposing the $(n-1)$ dimensional space into $(n-1) / 2$ 2-D subspaces, using either real decoupling transformations or symmetrical component approach [2], [3]. Each of the available $2^{n}$ voltage space vectors of an $n$-phase voltage source inverter (VSI) appears simultaneously in all such 2-D planes $\left(d_{1}-q_{1}, d_{2}-q_{2}\right.$, etc.). Selection of the active VSI voltage vectors in all the available studies follows the principle of [4], where it has been established that one needs to apply $(n-1)$ active vectors in order to generate sinusoidal output voltages. These are selected as the active vectors neighboring the reference space vector in the first $\left(d_{1}-q_{1}\right)$ plane. Each of the $(n-1) / 2$ planes will contain certain low-order harmonics and further development of the SVPWM depends on the type of the multiphase machine and on the number of machines controlled from a single VSI supply.

In a single-motor multiphase drive with sinusoidal MMF distribution, the requirement is to generate only sinusoidal output voltages. Hence, the reference voltage space vector is nonzero only in the $d_{1}-q_{1}$ plane and the SVPWM scheme has to ensure that zero average voltage space vector is applied in all the other planes, so that undesirable low order harmonics do not appear in the output. This is most easily done utilizing the analytical expressions for computation of the application times of the active vectors, such as those developed in [5]-[7] for a five-phase VSI, [8] for a seven-phase VSI, and [9] for a nine-phase VSI.

In a single-motor multiphase drive with concentrated stator winding machine it is desirable to utilize higher stator current harmonic injection for the purpose of the torque enhancement. The odd harmonics below the phase number $n$ can be used and one output voltage harmonic per additional plane $\left(d_{2}-q_{2}, d_{3}-q_{3}, \ldots, d_{(n-1) / 2}-q_{(n-1) / 2}\right)$ can be utilized. Thus, the reference voltage space vectors now have nonzero values in all the planes. If the active space vectors are selected on the basis of the reference in the $d_{1}-q_{1}$ plane, then the achievable voltages in all the other planes are automatically limited [10], [11]. This is, however, not the problem in this case since voltage references in all the other planes are considerably smaller than the reference in the $d_{1}-q_{1}$ plane. The calculation 
of the application times of the active vectors is typically done by solving online a system of equations that relates the axis components of the selected VSI space vectors with the corresponding components of the references [10], [11]. This imposes a significant computational burden on the DSP and makes this approach rather complicated for implementation purposes. Some improvements with regard to the implementation complexity have been reported in [12]. A different method, which relies on summation of device turn-on times for creating the desired references in the two planes, but still considers in the initial vector selection only neighboring vectors in the $d_{1}-q_{1}$ plane, has been developed in [13]. A general approach to find the dwell times, once when the active $(n-1)$ vectors have been selected, has been developed in [14] and further successfully applied to control of a five-phase brushless dc machine with third harmonic injection in [15].

The third and the most demanding situation that can arise is the multimotor series-connected multiphase drive system with a single multiphase VSI supply, where the machines are with sinusoidal MMF distribution. Assuming that the VSI phase number is an odd prime number, $(n-1) / 2 n$-phase machines can be connected in series using an appropriate phase transposition [16]. The supply comes from a single $n$-phase VSI and the independent control of the machines is made possible thanks to the phase transposition introduced in the series connection [16]. From the point of view of the PWM, this corresponds to the situation where there is one reference voltage space vector in each plane. Magnitudes and frequencies of these $(n-1) / 2$ reference voltage space vectors are completely independent one from the other, in contrast to the harmonic injection case discussed above. Hence, taking the two-motor five-phase drive system [17] as an example, the situation may arise where the magnitude of the reference in the $d_{2}-q_{2}$ plane (reference voltage of the second machine) is considerably larger than the reference in the $d_{1}-q_{1}$ plane (reference voltage of the first machine). The existing SVPWM methods, with vector selection based on the reference in the $d_{1}-q_{1}$ plane, are unable to cope with such a situation. Hence, the experimental studies on two-motor series-connected five-phase and six-phase drive systems with a single VSI supply [17]-[20] have all utilized carrier-based PWM, where desired inverter modulating signals are generated in a straightforward manner [21].

The only attempt to develop an SVPWM method for the five-phase two-motor structure, using the concept of multiple 2-D subspaces, has been described in [22]. It was suggested to select in each of the two planes, completely independently, a set of four active space vectors neighboring the corresponding reference. Then it becomes possible to create two voltage space vector references independently, using the same approach and the same analytical expressions as for the case of purely sinusoidal output voltage generation. However, the application of the selected vectors was done in such a way that $d_{1}-q_{1}$ plane reference voltage was applied in one switching period, while the $d_{2}-q_{2}$ plane voltage reference was applied in the next switching period. Such an approach to realization of the two space vector voltage references has automatically restricted the available fundamental for each of the two machines to only $50 \%$ of its maximum value for the given dc-link voltage (since only every second period is used for application of the voltage to any of the two machines).

An application where the five-phase two-motor drive system may find its place in the future is the two-motor winder system [17], [18]. In such a constant power application, while one machine runs at low speed (low voltage and frequency) with high torque (current), the other machine runs at high speed (high voltage and frequency) and low torque (low current). Voltage/current requirements continuously change during the rewinding and the situation is reversed at the end of the process [17], [18]. Hence, the two voltage references can be of both high frequency (high magnitude) and of low frequency (low magnitude), but never simultaneously. In such a case the SVPWM method of [22] is not adequate since it essentially requires practically doubling of the dc bus voltage (compared to a single-motor drive), while simultaneously disregarding actual operating states of the machines (and therefore effectively underutilizing the available dc bus voltage).

The aim of this paper is to demonstrate development of an alternative SVPWM scheme, which can be utilized for both single-motor multiphase drives with higher harmonic injection and for series-connected multiphase multimotor drives. The basic idea is the selection of a total of $(n-1)^{2} / 2$ active space vectors, i.e., $(n-1)$ active space vectors for each of the $(n-1) / 2 d-q$ planes, as proposed in [22]. However, in contrast to [22], the selected active space vectors are applied within a single switching period, thus avoiding the need for doubling the dc bus voltage. Furthermore, it is shown that the initial $(n-1)^{2} / 2$ active space vectors can be automatically reduced to the most appropriate $(n-1)$ active vectors (plus zero voltage space vectors) for the required reference voltage generation. These can be arranged in such a way that the switching frequency remains the same as in the existing SVPWM methods. Since the limiting on the realizable voltage in the $d_{2}-q_{2}$ (and $d_{3}-q_{3}$, etc., if applicable) plane(s) is not introduced by the voltage vector selection, it becomes possible to provide full utilization of the dc bus in the multimotor series-connected multiphase drives and, for the above described winder application, there is practically no need to increase the dc bus voltage above the value required for operation of a single machine. The implementation of the suggested SVPWM is simple and is therefore also well suited to the single-motor drives with higher harmonic injection. Detailed theoretical considerations are given for the SVPWM of a five-phase VSI, while the extension of the proposed SVPWM principle to higher phase numbers is discussed in the penultimate section of this paper. Experimental results, collected from the five-phase two-motor series-connected drive, are given to verify theoretical analysis.

\section{REVIEW OF A FIVE-PHASE VSI OPERATION}

By defining switching functions $m_{i}(i=a, b, c, d, e)$ for inverter legs with $m_{i}=1$ when upper switch is "on" and $m_{i}=0$ when it is "off," instantaneous values of phase-to-neutral voltages of a five-phase balanced load can be calculated using

$$
v_{i}=V_{\mathrm{dc}}\left[m_{i}-\frac{1}{5}\left(m_{a}+m_{b}+m_{c}+m_{d}+m_{e}\right)\right] \text {. }
$$




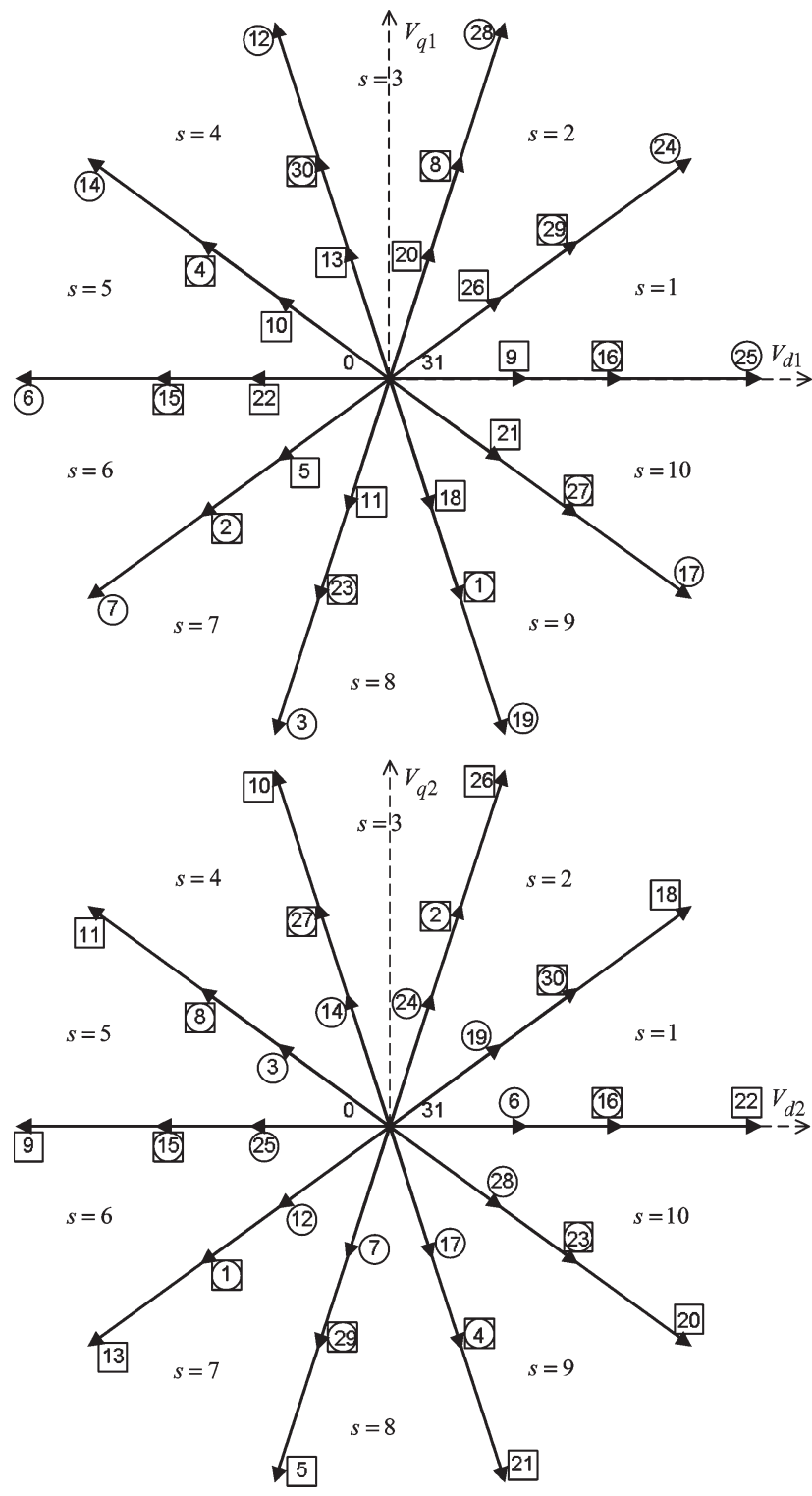

Fig. 1. Space vectors of a five-phase VSI in two 2-D subspaces.

During operation of a five-phase VSI, there are $2^{5}=32$ possible switching configurations depending on values of the switching functions $m_{i}$. Since load neutral point is isolated, a five-phase VSI can be described in two planes $\left(d_{1}-q_{1}\right.$ and $d_{2}-q_{2}$ ) by means of the following two space vectors [5], [11]:

$$
\begin{aligned}
& \bar{v}_{d_{1}-q_{1}}=\frac{2}{5}\left(v_{a}+v_{b} \bar{a}+v_{c} \bar{a}^{2}+v_{d} \bar{a}^{3}+v_{e} \bar{a}^{4}\right) \\
& \bar{v}_{d_{2}-q_{2}}=\frac{2}{5}\left(v_{a}+v_{b} \bar{a}^{2}+v_{c} \bar{a}^{4}+v_{d} \bar{a}+v_{e} \bar{a}^{3}\right)
\end{aligned}
$$

where $\bar{a}=\exp (j 2 \pi / 5)$. Application of (2) to phase-to-neutral voltages of (1) results in representation of phase-to-neutral voltage space vectors in the two subspaces (Fig. 1). There are 32 space vectors in each plane (30 active and two zero) and each space vector corresponds to one particular switching configuration. Space vectors are identified with decimal numbers in Fig. 1. By converting each decimal number into

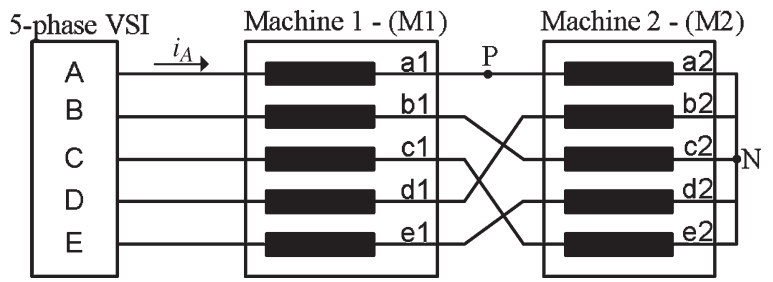

Fig. 2. Five-phase two-motor series-connected drive system.

a five-digit binary number, switching states that define a particular space vector are obtained (the most significant bit (MSB) of the binary number matches the value of the switching function $m_{a}$, the second MSB that of $m_{b}$, etc.). Active space vectors belong to three groups in accordance with their magnitudes - small, medium and large space vector groups. Their magnitudes are $\left|\bar{v}_{\mathrm{S}}\right|=4 / 5 \cos (2 \pi / 5) V_{\mathrm{dc}}$, $\left|\bar{v}_{\mathrm{M}}\right|=2 / 5 V_{\mathrm{dc}}$, and $\left|\bar{v}_{\mathrm{L}}\right|=4 / 5 \cos (\pi / 5) V_{\mathrm{dc}}$, respectively.

It can be observed from Fig. 1 that medium length space vectors of the $d_{1}-q_{1}$ plane map into medium length vectors in the $d_{2}-q_{2}$ plane. However, large vectors of the $d_{1}-q_{1}$ plane map into small vectors in the $d_{2}-q_{2}$ plane, while small vectors of the $d_{1}-q_{1}$ plane map into large vectors in the $d_{2}-q_{2}$ plane. Medium length vectors have the 4-1 switching configuration (four upper/lower and one lower/upper switch on), while both small and large space vectors have 3-2 switching configurations. The existing SVPWM methods [5]-[7], [11], [13] are based on selection of two large and two medium voltage space vectors in the $d_{1}-q_{1}$ plane, which neighbor the reference voltage space vector in a given sector. Since large vectors map into small vectors in $d_{2}-q_{2}$ plane, such a selection automatically limits the achievable maximum voltage reference in the second plane.

The second plane is usually used to control injection of the third harmonic voltage component when a five-phase VSI supplies a single machine with concentrated stator winding, since output voltage harmonics of the order $10 \nu \pm 1$ map into the $d_{1}-q_{1}$ plane, while harmonics of the order $10 \nu \pm 3$ belong to the $d_{2}-q_{2}$ plane $(\nu=0,1,2,3, \ldots)$. However, the second plane can also be used to control the second five-phase machine in the drive system with two series-connected machines supplied from a single VSI (Fig. 2). In this case both machines are with sinusoidal MMF distribution and the phase transposition in connection corresponds to phase shifting in calculation of the voltage space vectors in the $d_{2}-q_{2}$ plane in (2). Using vector space decomposition into two planes, the resulting equivalent circuit representation of the two-motor drive of Fig. 2 [23] is, from the VSI point of view (but not from the machines' point of view), identical to the one obtained for concentrated winding single-motor drive [24], [25]: the inverter has to generate nonzero voltage references in both planes. The important difference is however that for a concentrated winding machine the reference voltage in the second plane has a frequency firmly determined with the first plane reference; also, the second reference magnitude is only a small fraction of the first reference magnitude in steady state operation [11]. In the drive system of Fig. 2, two voltage references are completely independent from each other and can be of any magnitude [16], [17]. 


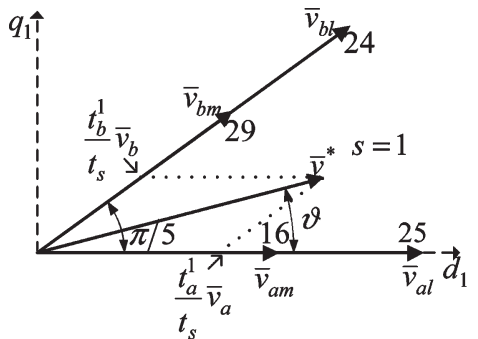

(a)

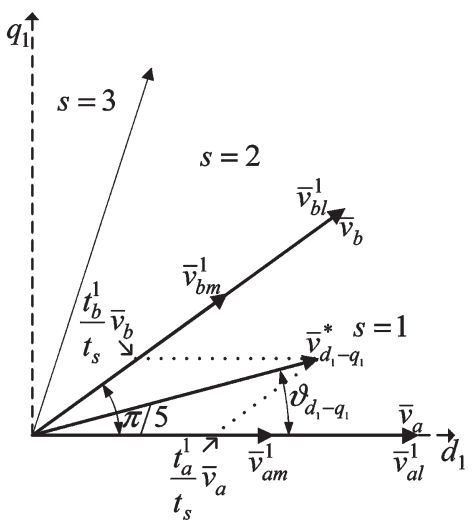

(c)

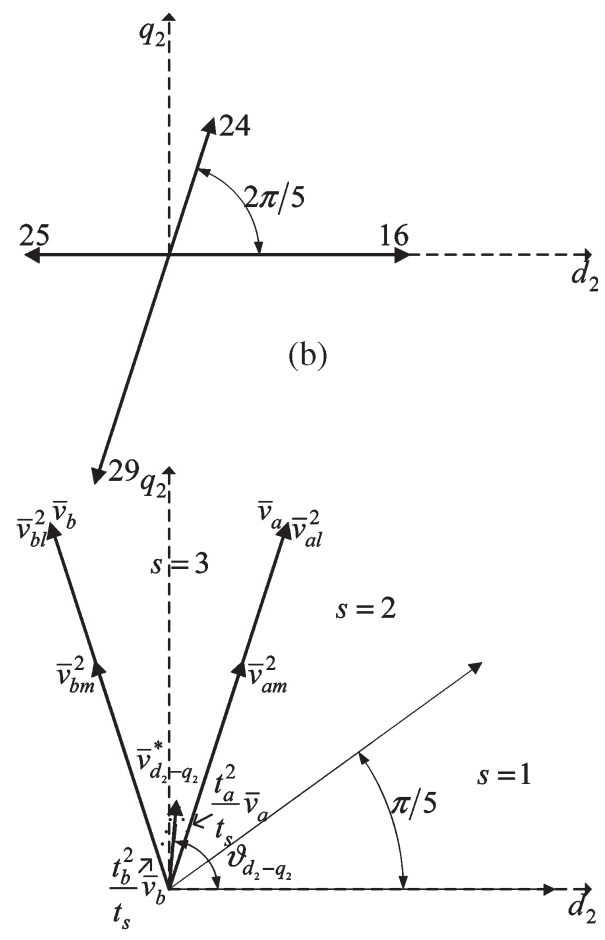

(d)

Fig. 3. SVPWM for five-phase VSI: (a) Calculation of active vector application times in the first $d-q$ plane and (b) position of selected active space vectors in the second $d-q$ plane; (c) and (d) two independent voltage space vector references in the two planes and associated active space vector selection independently in each of the two planes.

\section{SVPWM METhOdS FOR FIVE-PhASE VSIS}

A summary of the SVPWM methods for single-motor drives (initially with sinusoidal MMF and then with concentrated stator winding) is provided first. It is assumed that position of the reference(s) is known (this is simple to determine in open-loop $V / f$ drives and slightly more involved in currentcontrolled vector drives). The reference can then be obtained on average by applying a certain number of active space vectors for adequate time intervals, without saturating the VSI. Four active space vectors are required to generate sinusoidal voltages [4]-[7]. Suppose that the reference space vector in the first $d_{1}-q_{1}$ plane, $\bar{v}_{d_{1}-q_{1}}^{*}=\bar{v}^{*}$, is in sector $s=1$ [Fig. 3(a)]. Two neighboring large and two medium space vectors are selected. Times of application of active space vectors have to satisfy the following constraints in order to provide zero average applied voltage in the second $d_{2}-q_{2}$ plane, $\bar{v}_{d_{2}-q_{2}}^{*}=0$ [Fig. 3(b)]:

$$
\begin{aligned}
\left|\bar{v}_{\mathrm{M}}\right| t_{a m}+\left|\bar{v}_{\mathrm{L}}\right| t_{a l} & =\frac{\left|\bar{v}^{*}\right| \sin (s \pi / 5-\vartheta)}{\sin (\pi / 5)} t_{\mathrm{s}} \\
\left|\bar{v}_{\mathrm{M}}\right| t_{a m}-\left|\bar{v}_{\mathrm{S}}\right| t_{a l} & =0 \\
\left|\bar{v}_{\mathrm{M}}\right| t_{b m}+\left|\bar{v}_{\mathrm{L}}\right| t_{b l} & =\frac{\left|\bar{v}^{*}\right| \sin [\vartheta-(s-1) \pi / 5]}{\sin (\pi / 5)} t_{\mathrm{S}} \\
\left|\bar{v}_{\mathrm{M}}\right| t_{b m}-\left|\bar{v}_{\mathrm{S}}\right| t_{b l} & =0
\end{aligned}
$$

where $t_{\mathrm{s}}$ is the switching period, $\vartheta$ is the reference position and indices $a$ and $b$ are defined in Fig. 3(a). Total time of application of zero space vectors $t_{o}=t_{\mathrm{s}}-\left(t_{a l}+t_{a m}+t_{b l}+t_{b m}\right)$ is equally shared by zero space vectors $\bar{v}_{0}$ and $\bar{v}_{31}$. By solving (3) and (4), dwell times for active space vectors are

$$
\begin{aligned}
t_{a l} & =\frac{2 \sin (2 \pi / 5)}{V_{\mathrm{dc}}} \sin (s \pi / 5-\vartheta)\left|\bar{v}^{*}\right| t_{\mathrm{s}} \\
t_{a m} & =\frac{2 \sin (\pi / 5)}{V_{\mathrm{dc}}} \sin (s \pi / 5-\vartheta)\left|\bar{v}^{*}\right| t_{\mathrm{s}} \\
t_{b l} & =\frac{2 \sin (2 \pi / 5)}{V_{\mathrm{dc}}} \sin [\vartheta-(s-1) \pi / 5]\left|\bar{v}^{*}\right| t_{\mathrm{s}} \\
t_{b m} & =\frac{2 \sin (\pi / 5)}{V_{\mathrm{dc}}} \sin [\vartheta-(s-1) \pi / 5]\left|\bar{v}^{*}\right| t_{\mathrm{s}} .
\end{aligned}
$$

The maximum peak value of the output phase-to-neutral voltage in the linear region is $V_{\max }=1 /[2 \cos (\pi / 10)] \cong$ $0.526 V_{\mathrm{dc}}$ [5]-[7]. Switching pattern is a symmetrical PWM with two commutations for each inverter leg. The space vectors are applied in odd sectors using sequence $\left(\bar{v}_{0} \bar{v}_{a m} \bar{v}_{b l} \bar{v}_{a l} \bar{v}_{b m} \bar{v}_{31} \bar{v}_{b m} \bar{v}_{a l} \bar{v}_{b l} \bar{v}_{a m} \bar{v}_{0}\right)$, while the sequence is $\left(\bar{v}_{0} \bar{v}_{b m} \bar{v}_{a l} \bar{v}_{b l} \bar{v}_{a m} \bar{v}_{31} \bar{v}_{a m} \bar{v}_{b l} \bar{v}_{a l} \bar{v}_{b m} \bar{v}_{0}\right)$ in even sectors. Expressions (5) and (6) are simple for implementation and they satisfy all the needs if purely sinusoidal output is required.

If a concentrated winding machine is supplied, reference in the second $d-q$ plane, $\bar{v}_{d_{2}-q_{2}}^{*}$, could be of nonzero value [11]. In this case, four active space vectors $\bar{v}_{i}, \bar{v}_{j}, \bar{v}_{h}, \bar{v}_{k}$ should be properly selected from the set of available $30, \bar{v}_{1}, \bar{v}_{2}, \ldots, \bar{v}_{30}$. 


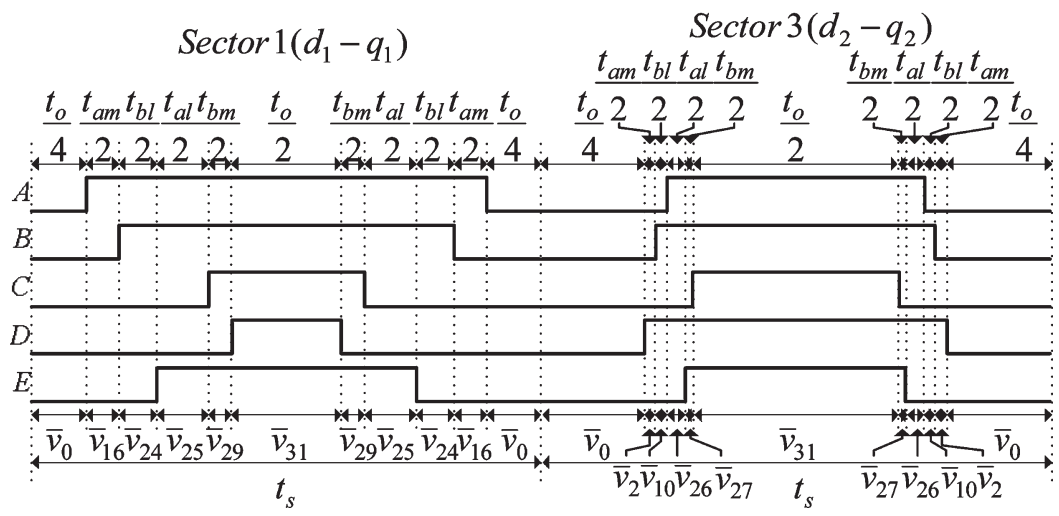

Fig. 4. Switching pattern obtained with SVPWM-Method 1.

The corresponding application times are now found by solving online the set of equations [2], [10], [11]

$$
\begin{aligned}
v_{i d_{1}} t_{i}+v_{j d_{1}} t_{j}+v_{h d_{1}} t_{h}+v_{k d_{1}} t_{k} & =v_{d_{1}}^{*} t_{\mathrm{s}} \\
v_{i q_{1}} t_{i}+v_{j q_{1}} t_{j}+v_{h q_{1}} t_{h}+v_{k q_{1}} t_{k} & =v_{q_{1}}^{*} t_{\mathrm{s}} \\
v_{i d_{2}} t_{i}+v_{j d_{2}} t_{j}+v_{h d_{2}} t_{h}+v_{k d_{2}} t_{k} & =v_{d_{2}}^{*} t_{\mathrm{s}} \\
v_{i q_{2}} t_{i}+v_{j q_{2}} t_{j}+v_{h q_{2}} t_{h}+v_{k q_{2}} t_{k} & =v_{q_{2}}^{*} t_{\mathrm{s}}
\end{aligned}
$$

where $i, j, h, k$ are in the range $1 \div 30$, and the total time of application of zero space vectors $t_{o}=t_{\mathrm{s}}-\left(t_{i}+t_{j}+t_{h}+t_{k}\right)$ can be shared by zero space vectors $\bar{v}_{0}$ and $\bar{v}_{31}$. Here, indices $d_{1}, q_{1}, d_{2}, q_{2}$ stand for projections of the space vectors along the four axes of the 4-D space.

As far as the two-motor drive of Fig. 2 is concerned, application of (7) is feasible, provided that the most appropriate four active space vectors are selected. However, active space vector selection based on reference in the $d_{1}-q_{1}$ plane is now inappropriate since references in the two planes are unrelated, can be anywhere in the planes, can have an arbitrary ratio of magnitudes and both magnitudes can be large. A general method to determine the proper four space vectors has not been found yet, and the calculations based on (7) could be very time consuming for online implementation [2], [10]-[12].

\section{Multifrequency Output Voltage Generation}

\section{A. SVPWM-Method 1}

The SVPWM method of [22] is reviewed first, since the initial active space vector selection considered in this paper is based on the same approach. Since there are two independent voltage references in two planes, it is suggested in [22] to select for each of the two references four neighboring active space vectors [Fig. 3(c) and (d)], in the same manner as it has been done in the preceding section for a single-motor drive. Two medium and two large vectors are selected in each plane. This means that, in general, there are eight active space vectors that need to be imposed. In terms of the first $d-q$ plane there are four medium, two large and two small vectors. However, one of the medium length vectors selected in the two planes may be the same. This depends on the two space vector references and means that the total number of selected different active space vectors is either seven or eight.

Two independent space vector modulators are further utilized to realize required two voltage space vector references, with dwell times calculated independently in the two planes using (5), (6). The modulators impose the required references in a sequential manner, as illustrated in Fig. 4 where switching pattern is shown for two consecutive switching periods for the references positioned as in Fig. 3(c) and (d) (space vectors that are included in the switching pattern are those shown in Fig. 1, where numbers inside a circle/square correspond to the active space vector from the first/second plane responsible for voltage generation for the first/second machine). Fig. 4 is drawn to scale for one particular pair of the voltage space vector references in the two planes in one instant in time (this pair of references is used further on in all considered PWM schemes for illustrative purposes): $\left|\bar{v}_{d_{1}-q_{1}}^{*}\right|=0.3 V_{\mathrm{dc}}, \vartheta_{d_{1}-q_{1}}=15^{\circ}$ (i.e., in the first sector) and $\left|\bar{v}_{d_{2}-q_{2}}^{*}\right|=0.1 V_{\mathrm{dc}}, \vartheta_{d_{2}-q_{2}}=85^{\circ}$ (i.e., in the third sector). However, since in each switching period only one reference is imposed while simultaneously zeroing the applied voltage in the other plane, the effective values of the output voltages will be one half of the given reference values. This simultaneously means that the effective realizable reference in both planes is restricted to only $50 \%$ of the maximum value obtainable for the given dc link voltage, regardless of the reference value in the other plane. Thus, even if the first machine requires zero voltage and there is potentially the whole dc bus voltage available for the control of the second machine, only half of the full dc voltage can be applied due to the SVPWM method nature. This is illustrated in Fig. 5 where the checkered area represents realizable pairs of references in the two $d-q$ planes. Each reference is restricted to at most $0.263 V_{\mathrm{dc}}$ (i.e., $50 \%$ of the maximum value), regardless of the value of the other reference.

An additional disadvantage is that, since the switching pattern repeats every two periods, the first harmonic sideband appears around one half of the switching frequency.

Further development aims at removing the limitations of the sequential approach of [22]. Because the series-connected two-motor drive is aimed at winder applications, it is required to provide full $\mathrm{dc}$ bus utilization by respecting the operating conditions of the two machines in the SVPWM scheme. In other 


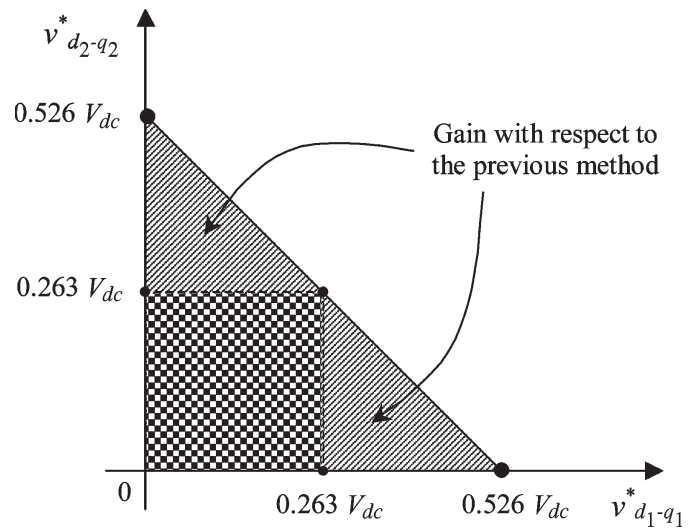

Fig. 5. Illustration of the realizable references with the existing method of [22] (checkered area) and the perceived gain obtained with the method proposed in Section IV-B (shaded areas).

words, the aim is to expand the operating region in Fig. 5 so that it includes the shaded areas (in addition to the checkered area) and thus improve substantially dc bus utilization for all operating conditions.

\section{B. SVPWM-Method 2}

In the first method each reference is created in one switching period using four active vectors in the given plane. However, it is observed that, from the point of view of the application of any of the two space vector voltage references, application of the other reference is identical to the zero space vector application, since (5), (6) zero the average voltage in the other plane. This opens up a possibility of replacing zero space vectors with active space vectors from the other plane. Therefore, instead of using two consecutive periods for voltage control in the two planes, only one switching period can be used. It can be considered as consisting of two subperiods $t_{\mathrm{s}}^{1}, t_{\mathrm{s}}^{2}\left(t_{\mathrm{s}}=t_{\mathrm{s}}^{1}+t_{\mathrm{s}}^{2}\right)$ that are proportional to the magnitudes of reference space vectors in the two planes, according to

$$
t_{\mathrm{s}}^{1}=\frac{\left|\bar{v}_{d_{1}-q_{1}}^{*}\right|}{\left|\bar{v}_{d_{1}-q_{1}}^{*}\right|+\left|\bar{v}_{d_{2}-q_{2}}^{*}\right|} t_{\mathrm{s}}, \quad t_{\mathrm{s}}^{2}=\frac{\left|\bar{v}_{d_{2}-q_{2}}^{*}\right|}{\left|\bar{v}_{d_{1}-q_{1}}^{*}\right|+\left|\bar{v}_{d_{2}-q_{2}}^{*}\right|} t_{\mathrm{s}} .
$$

Application times for both sets of four active vectors are still calculated individually for two references using (5) and (6)

$$
\begin{aligned}
t_{a l}^{1} & =\frac{2 \sin (2 \pi / 5)}{V_{\mathrm{dc}}} \sin \left(s^{1} \pi / 5-\vartheta_{d_{1}-q_{1}}\right)\left|\bar{v}_{d_{1}-q_{1}}^{*}\right| t_{\mathrm{s}} \\
t_{a m}^{1} & =\frac{2 \sin (\pi / 5)}{V_{\mathrm{dc}}} \sin \left(s^{1} \pi / 5-\vartheta_{d_{1}-q_{1}}\right)\left|\bar{v}_{d_{1}-q_{1}}^{*}\right| t_{\mathrm{s}} \\
t_{b l}^{1} & =\frac{2 \sin (2 \pi / 5)}{V_{\mathrm{dc}}} \sin \left[\vartheta_{d_{1}-q_{1}}-\left(s^{1}-1\right) \pi / 5\right]\left|\bar{v}_{d_{1}-q_{1}}^{*}\right| t_{\mathrm{s}} \\
t_{b m}^{1} & =\frac{2 \sin (\pi / 5)}{V_{\mathrm{dc}}} \sin \left[\vartheta_{d_{1}-q_{1}}-\left(s^{1}-1\right) \pi / 5\right]\left|\bar{v}_{d_{1}-q_{1}}^{*}\right| t_{\mathrm{s}} \\
t_{a l}^{2} & =\frac{2 \sin (2 \pi / 5)}{V_{\mathrm{dc}}} \sin \left(s^{2} \pi / 5-\vartheta_{d_{2}-q_{2}}\right)\left|\bar{v}_{d_{2}-q_{2}}^{*}\right| t_{\mathrm{s}} \\
t_{a m}^{2} & =\frac{2 \sin (\pi / 5)}{V_{\mathrm{dc}}} \sin \left(s^{2} \pi / 5-\vartheta_{d_{2}-q_{2}}\right)\left|\bar{v}_{d_{2}-q_{2}}^{*}\right| t_{\mathrm{s}}
\end{aligned}
$$

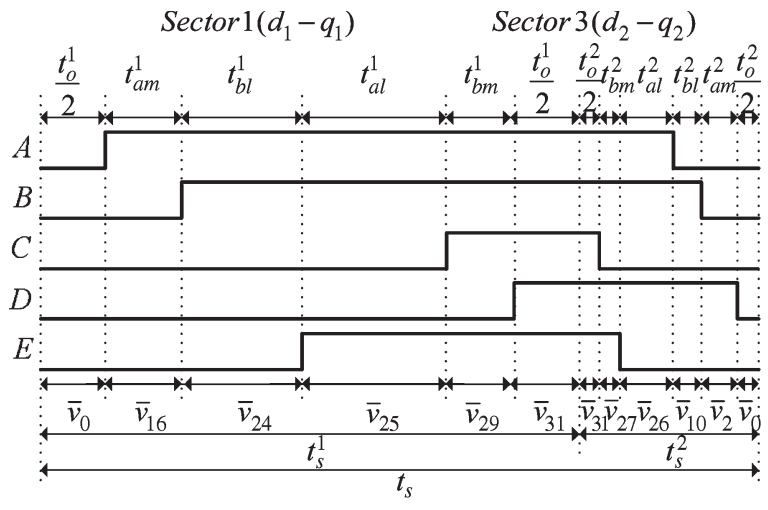

Fig. 6. Switching pattern obtained with SVPWM-Method 2.

$$
\begin{aligned}
t_{b l}^{2} & =\frac{2 \sin (2 \pi / 5)}{V_{\mathrm{dc}}} \sin \left[\vartheta_{d_{2}-q_{2}}-\left(s^{2}-1\right) \pi / 5\right]\left|\bar{v}_{d_{2}-q_{2}}^{*}\right| t_{\mathrm{s}} \\
t_{b m}^{2} & =\frac{2 \sin (\pi / 5)}{V_{\mathrm{dc}}} \sin \left[\vartheta_{d_{2}-q_{2}}-\left(s^{2}-1\right) \pi / 5\right]\left|\bar{v}_{d_{2}-q_{2}}^{*}\right| t_{\mathrm{s}}
\end{aligned}
$$

where superscripts 1 and 2 apply to planes $d_{1}-q_{1}$ and $d_{2}-q_{2}$, respectively. The zero space vector dwell times now become

$$
\begin{aligned}
t_{o}^{1}= & t_{\mathrm{s}}^{1}-\left(t_{a l}^{1}+t_{a m}^{1}+t_{b l}^{1}+t_{b m}^{1}\right) \\
t_{o}^{2}= & t_{\mathrm{s}}^{2}-\left(t_{a l}^{2}+t_{a m}^{2}+t_{b l}^{2}+t_{b m}^{2}\right) \\
t_{o}= & t_{o}^{1}+t_{o}^{2}=t_{\mathrm{s}}-\left(t_{a l}^{1}+t_{a m}^{1}+t_{b l}^{1}+t_{b m}^{1}\right) \\
& \quad-\left(t_{a l}^{2}+t_{a m}^{2}+t_{b l}^{2}+t_{b m}^{2}\right) .
\end{aligned}
$$

It follows from (15) that the two references can be created within one switching period as long as the zero space vector application time does not become negative. Total time of application of zero space vectors can be shared between two subperiods in accordance with the reference space vector magnitudes.

The switching pattern now becomes as illustrated in Fig. 6 . By introducing variable subperiods as functions of the individual voltage reference magnitudes in the two planes it becomes possible to fully utilize the available dc bus voltage. In the context of the series connected two-motor drive this means that one machine may receive full voltage (i.e., run at rated speed) if the other machine is at standstill (i.e., with voltage requirement close to zero), and vice versa. It should be noted that although the illustration in Fig. 6 applies to the references positioned in sectors 1 and 3 of the $d_{1}-q_{1}$ and $d_{2}-q_{2}$ planes, respectively, it can be shown that the same kind of the switching pattern can be obtained for all possible combinations of the sectors $s^{1}, s^{2}$ in which the two references are $(10 \times 10=100$ sector combinations).

This approach to SVPWM (Fig. 6) is easy to understand and it also clearly shows the application of eight active space vectors in one switching period, while avoiding the voltage harmonics around one half of the switching frequency and preserving the same switching frequency as in Fig. 4. Control over both machines is now performed over one switching period, in contrast to method 1 where two consecutive periods were used. 


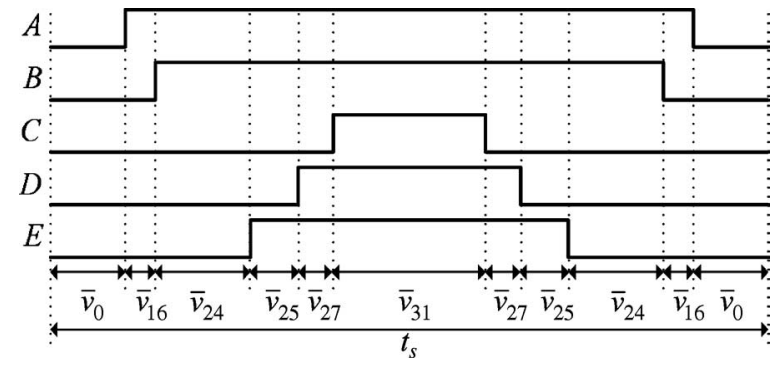

Fig. 7. Switching pattern obtained with SVPWM-Method 3.

\section{SVPWM-Method 3}

As shown in the previous section, introduction of two subperiods of variable durations enables operation with any two voltage references that satisfy condition (15). The use is made of the fact that each of the two voltage space vector references is applied in such a manner that the average voltage in the other plane is kept at zero. Hence, active vectors used to generate reference in one plane can be used to replace zero vectors in the other plane. There are all together eight active vectors that are applied in a switching period and the switching pattern is asymmetrical, this being the cause of potential difficulties in implementation (as explained in the following section). Hence, the method of the previous section is modified as follows.

The average voltage of an inverter leg over the switching period is a function of only the duty cycle applied to that leg. Thus, if switching pattern of Fig. 6 is centered with respect to the midpoint of the switching period, while keeping the on/off ratio the same for each leg, the average voltage of each inverter leg will stay the same. However, this will result in symmetrical PWM pattern that is easy to implement using standard DSP PWM units. Hence, the following modifications are introduced. To preserve the same values of the average leg voltages as in Fig. 6, the application times of active space vectors from both planes, determined independently by the two modulators, are summed on a per-leg basis. Calculation of application times of active space vectors is the same as before and relies on (9)-(12). Since subperiods do not exist any more, calculations (8), (13) and (14) are omitted. Finally, total application time of zero space vectors is shared equally between two zero space vectors, thus taking care of the centering and providing a symmetrical PWM. The switching pattern, obtained with method 2 (Fig. 6), becomes as shown in Fig. 7 after the described modifications.

It can be observed from Fig. 7 that, after centering, only four instead of eight active space vectors are applied. The four vectors are in this case a subset of the original eight vectors in Fig. 6. In general, however, this is not necessarily the case and this is a property of the SVPWM scheme introduced by the centering operation. A detailed study has shown that the applied set of four active vectors will always contain two medium length vectors. Referencing the vector lengths with respect to the first $d_{1}-q_{1}$ plane, the other two can however be both large (in principle, if reference in $d_{1}-q_{1}$ is larger than the reference in the $d_{2}-q_{2}$ plane), both small (in principle, if reference in $d_{2}-q_{2}$ is larger than the reference in the $d_{1}-q_{1}$ plane) or there may be one large and one small (in principle, when both references
TABLE I

Active Vectors Utilized in Switching Pattern of Fig. 7 FOR A FEW RefERENCE Pairs (SeCOND Row Illustrated IN Fig. 7)

\begin{tabular}{|c|c|c|c|c|}
\hline \multicolumn{2}{|c|}{$d_{1}-q_{1}$ reference } & \multicolumn{2}{|c|}{$d_{2}-q_{2}$ reference } & Active vectors \\
\hline magnitude & phase & magnitude & phase & \\
\hline $0.5 V_{d c}$ & $15^{\circ}$ & 0 & - & $16,24,25,29$ \\
\hline $0.3 V_{d c}$ & $15^{\circ}$ & $0.1 V_{d c}$ & $85^{\circ}$ & $16,24,25,27$ \\
\hline $0.2 V_{d c}$ & $15^{\circ}$ & $0.2 V_{d c}$ & $85^{\circ}$ & $\mathbf{8 , 2 4 , 2 6 , 2 7}$ \\
\hline $0.2 V_{d c}$ & $5^{\circ}$ & $0.2 V_{d c}$ & $110^{\circ}$ & $\mathbf{8 , 2 4 , 2 5 , 2 7}$ \\
\hline $0.2 V_{d c}$ & $30^{\circ}$ & $0.2 V_{d c}$ & $75^{\circ}$ & $16,24,26,27$ \\
\hline $0.1 V_{d c}$ & $15^{\circ}$ & $0.3 V_{d c}$ & $85^{\circ}$ & $\mathbf{8 , 1 0 , 2 6 , 2 7}$ \\
\hline 0 & - & $0.5 V_{d c}$ & $85^{\circ}$ & $2,10,26,27$ \\
\hline
\end{tabular}

are of similar/same magnitudes and in different positions in the planes).

As an example, Table I illustrates resulting four active space vectors that will be applied for various combinations of magnitudes and phases of the two space vector references. For all cases illustrated in Table I, two space vector voltage references are in the first sector and the third sector of the two planes in Fig. 1, respectively. Hence, the initially selected eight active space vectors, in accordance with Fig. 6, are always the same and are 16, 24, 25, 29 and 2, 10, 26, 27. Depending on the magnitude and phase of reference space vectors in the two planes, the resulting four active space vectors, applied after the centering, will differ although the input set of eight active space vectors is always the same since reference vectors are always in the same sectors. Space vectors in bold font are those that will be ultimately applied as one of the four active vectors, although they were not initially present in the set of the eight vectors.

Another very important difference, brought in by the centering operation, is the change in the total time of zero vector application. Although the switching patterns in Figs. 6 and 7 apply to exactly the same pair of references, it is easy to see that the total application time of zero vectors (vectors 0 and 31 ) in Fig. 7 is considerably longer than in Fig. 6. This enables a further improvement in the dc bus voltage utilization in the linear modulation region, when compared to the limit of the method 2 illustrated in Fig. 5. It has already been established in [21] that, with carrier-based PWM, the limit of the linear modulation region is reached when both references simultaneously take the value of $0.325 V_{\mathrm{dc}}$. Exactly the same value has been obtained by simulation for the SVPWM method of this section. A detailed simulation study has shown that the dc bus utilization is now governed with an extended region (compared to method 2). This is illustrated in Fig. 8. More detailed considerations will be reported in the near future.

Since the limits of the linear modulation region are now as shown in Fig. 8, the applied control scheme (vector control, for example) should ensure operation in the linear PWM region without saturation of the VSI. In practice this means that voltage reference limiting in the two planes has to be mutually dependent. More detailed considerations are beyond the scope of this paper.

\section{IMPLEMENTATION}

The SVPWM methods of Sections IV-B and IV-C have been implemented in a TMS320F2812 DSP, which is used to control 


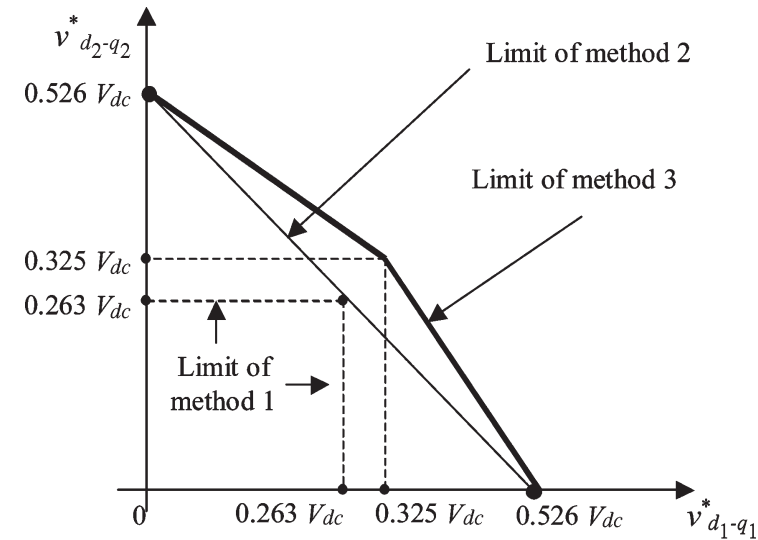

Fig. 8. Comparison of operating limits of all three SVPWM methods discussed in Section IV.

a custom-built multiphase VSI supplying two five-phase machines in series connection. Since there are five inverter legs to control, both event managers (A and B) of the DSP are utilized and proper synchronization of the DSP timers is performed. At the implementation stage, two independent SVPWM modulators are developed (one in each of the two planes), based on the theoretical considerations presented in the preceding part of this paper. Due to the use of standard DSP PWM hardware units that operate based on carrier-comparison principle, outputs of each modulator can be considered as modulating signals that will be loaded to compare registers of the DSP. Value of these signals is subsequently compared with the DSP timer values (carrier) in order to define the shape of the output PWM signals. Therefore, although the introduced PWM methods are based on the space vector theory, notions like modulation and carrier signal play an important role with regard to implementation using DSPs.

Although method 2 makes the full dc bus voltage available to one machine if the other one does not require voltage (and vice versa), it is not convenient for practical DSP-based implementation, since it involves two variable time subperiods, one of which may approach zero value. Problems arise due to the need to handle very low subperiod values, since the resolution of the DSP counters/timers is limited, and due to the inability to use the existing DSP PWM units in a standard way (since different carrier up/down slopes are required). The need to calculate variable subperiods also increases computational complexity. Setting the DSP counters/timers to count in continuous "up" mode generates saw-tooth shaped carrier, the period of which is controlled alternatively by calculated subperiods. Further action on DSP PWM unit output polarity and additional software modifications make the implementation of method 2 possible using standard DSPs, at the expense of an increased computational complexity. The resulting PWM pattern, generated by the DSP for the same conditions used throughout this paper, is shown in Fig. 9. The effect of two different subperiods, separated by the dashed vertical line in Fig. 9, is clearly visible, as is the application of different active space vectors at the beginning of the PWM pattern (vectors from the first plane) and at the end of the PWM pattern (vectors from the second plane). It should be noted that the experimental result in Fig. 9 is identical to the

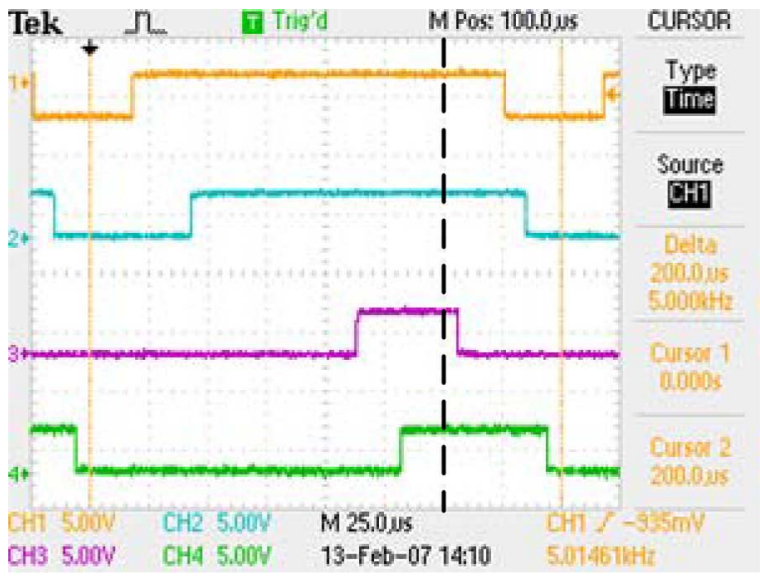

Fig. 9. PWM signals-Method 2 (inverter legs A, B, C, and D).

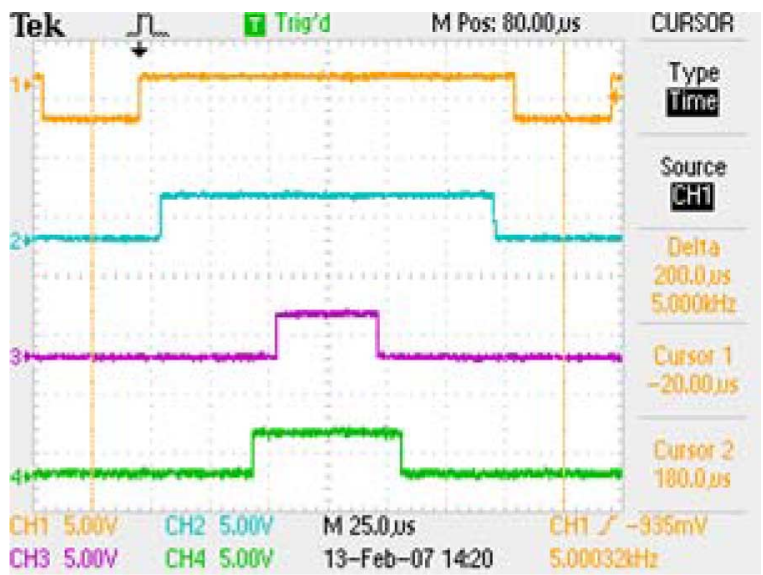

Fig. 10. PWM signals-Method 3 (inverter legs A, B, C, and D).

theoretical study displayed in Fig. 6 (both apply to the same voltage space vector reference values).

The aforementioned implementation-related difficulties are eliminated if method 3 is utilized. Centering of pulses generated with method 2 is obtained by resetting DSP counters/timers to operate in continuous up/down mode, thus creating triangular carrier signal. The resulting modulating signals for each inverter leg are obtained based on the output of each modulator and considering equal zero space vector placement. This effectively places active parts of PWM pulses from Fig. 9 in the middle of the switching period, as shown in Fig. 10. At the same time original eight active vectors are replaced with the four most appropriate active space vectors that will satisfy the required average voltage generation in each of the two planes.

A schematic illustration of the implementation process is shown in Fig. 11, where the segments within the dotted-line box represent actual software implementation of method 3 based on two SVPWM modulators, while the rest of the figure corresponds to the principle of operation of the DSP PWM units. Voltage requirement of any of the two machines is in this manner analyzed in ten sectors in the relevant $\left(d_{1}-q_{1}\right.$ or $\left.d_{2}-q_{2}\right)$ plane. Since the two references are mutually independent and can be positioned in any sector of the relevant plane, one in this manner avoids the need to deal with all $10 \times 10=100$ combinations of 2-D sectors that can take place. Experimental results, 


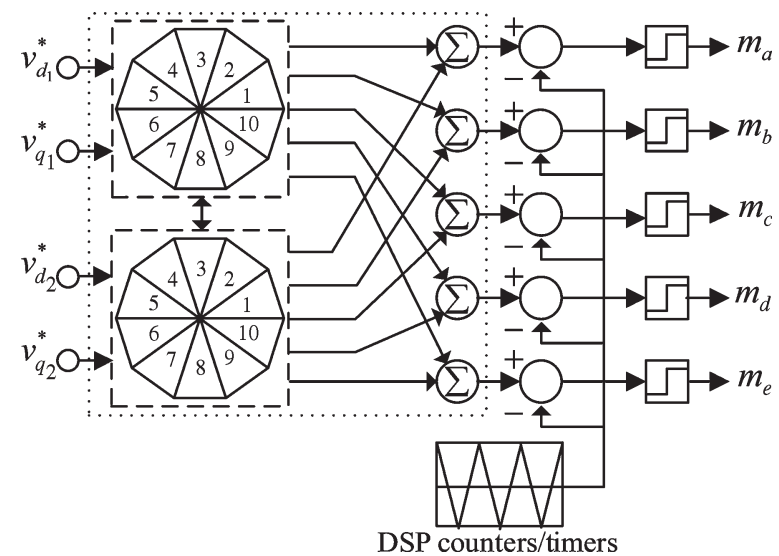

Fig. 11. Signal flow in the DSP-based implementation of the developed SVPWM method of Section IV-C.

presented in the following section, prove that new sequences of four active vectors, created in the described manner, effectively synthesize commanded voltage references for both machines.

Finally, it is worth noting that the PWM signal sequence, illustrated in Figs. 6, 7 and 9, 10 utilizes both available zero space vectors. This is so, first of all, because the goal was here to realize continuous PWM. Second, such a sequence is also a result of the desire to achieve a simple DSP implementation, yielding a PWM pattern that can be obtained with the existing DSP PWM units. Therefore, the simplest way to realize centering, shown in Figs. 7 and 10, is to allow the DSP timer/counter units to perform this task.

\section{EXPERIMENTAL RESULTS}

For the purpose of the experimental verification, method 3 is tested using a two-motor five-phase series-connected induction motor drive. Dynamics of this drive system under vector control conditions have been examined in [17] and [18], where full decoupling of the control of the two machines has been demonstrated (and ramp-comparison current control was implemented in conjunction with the carrier-based PWM). Since the goal here is to test the SVPWM scheme, the simplest $V / f=$ const. control principle is utilized and the motors operate under no-load conditions in steady state. The experimental rig is illustrated in Fig. 12 and the $50 \mathrm{~Hz}$ five-phase induction motors are those used previously in [17], [18], and [23].

Measured and low-pass filtered phase voltages (time-domain waveforms and spectra; filter cutoff frequency is $1.6 \mathrm{kHz}$ ) are shown in what follows, for a few operating conditions. The measured voltages are, with reference to Fig. 2, inverter phase voltage, $v_{\mathrm{AN}}$, and phase voltage of $\mathrm{M} 2, v_{\mathrm{PN}}$. Inverter current $i_{\mathrm{A}}$ waveform is also included for one case. Reference voltage of M1 is of frequency $f_{1}$ and is in the $d_{1}-q_{1}$ plane, while reference voltage of M2 is of frequency $f_{2}$ and it appears in the $d_{2}-q_{2}$ plane. In accordance with the steady state equivalent circuit representation for this drive configuration [23], measured inverter phase voltage will contain two different frequency components that will correspond to the two sets of references in terms of both magnitude and frequency. On the other hand, phase voltage of M2 will contain a component at frequency $f_{2}$ that

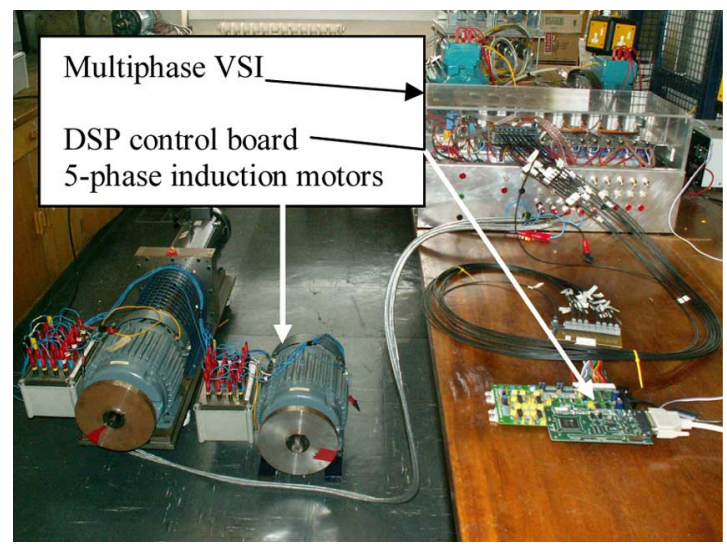

Fig. 12. Experimental setup.

will be slightly smaller than the corresponding component in the inverter phase voltage, due to the additional voltage drop at M1 at frequency $f_{2}$. M2 voltage will also have a small component at frequency $f_{1}$, this being the additional voltage drop at M2 (on stator leakage impedance) due to the flow of the currents of frequency $f_{1}$ through M2 [23].

The first set of measured voltages is shown in Fig. 13. The voltage reference magnitudes of the two machines are governed with frequencies $f_{1}=33 \mathrm{~Hz}$ and $f_{2}=16 \mathrm{~Hz}$. The inverter voltage spectrum contains two corresponding components of app. 80 and $40 \mathrm{~V}$, respectively. Most of the $16 \mathrm{~Hz}$ component also appears in the spectrum of M2 voltage, while the component at $33 \mathrm{~Hz}$ is $6 \mathrm{~V}$, since most of this frequency component is the voltage at $\mathrm{M} 1$.

Operating conditions of Fig. 13 correspond to the case when the voltage reference magnitude in the $d_{1}-q_{1}$ plane is twice the voltage reference magnitude in the $d_{2}-q_{2}$ plane. Hence, the applied four active space vectors are dominated by the vectors neighboring the reference in the $d_{1}-q_{1}$ plane, as discussed in conjunction with Table I. In the second operating condition, illustrated in Fig. 14, the situation is different. The frequencies are now $f_{1}=20 \mathrm{~Hz}$ and $f_{2}=25 \mathrm{~Hz}$, which means that the magnitudes of the two voltage space vector references are close to one another. Under these conditions the applied four active space vectors are never from the same plane and may involve a vector that is not neighboring any of the two references (middle portion of Table I). The components at 20 and $25 \mathrm{~Hz}$ are now app. 50 and $60 \mathrm{~V}$, respectively, in the inverter voltage. Most of the $60 \mathrm{~V}$ appears on M2 (operating at $25 \mathrm{~Hz}$ ) while most of the $20 \mathrm{~Hz}$ appears on M1 (and the $20 \mathrm{~Hz}$ component at M2 is small, around $2.5 \mathrm{~V}$ ).

The last operating point, shown in Fig. 15, applies to the frequency pair $f_{1}=10 \mathrm{~Hz}$ and $f_{2}=40 \mathrm{~Hz}$. Since the voltage reference in the $d_{2}-q_{2}$ plane is now four times larger than the reference in the $d_{1}-q_{1}$ plane, the applied four active space vectors are dominated by the vectors neighboring the reference in the $d_{2}-q_{2}$ plane. This is from the point of view of the applied four active space vectors, the situation inverse to the one depicted in Fig. 13. The magnitudes of two frequency components in Fig. 15 are in full agreement with the applied $V / f=$ const. control law and the values already observed in Figs. 13 and 14. 

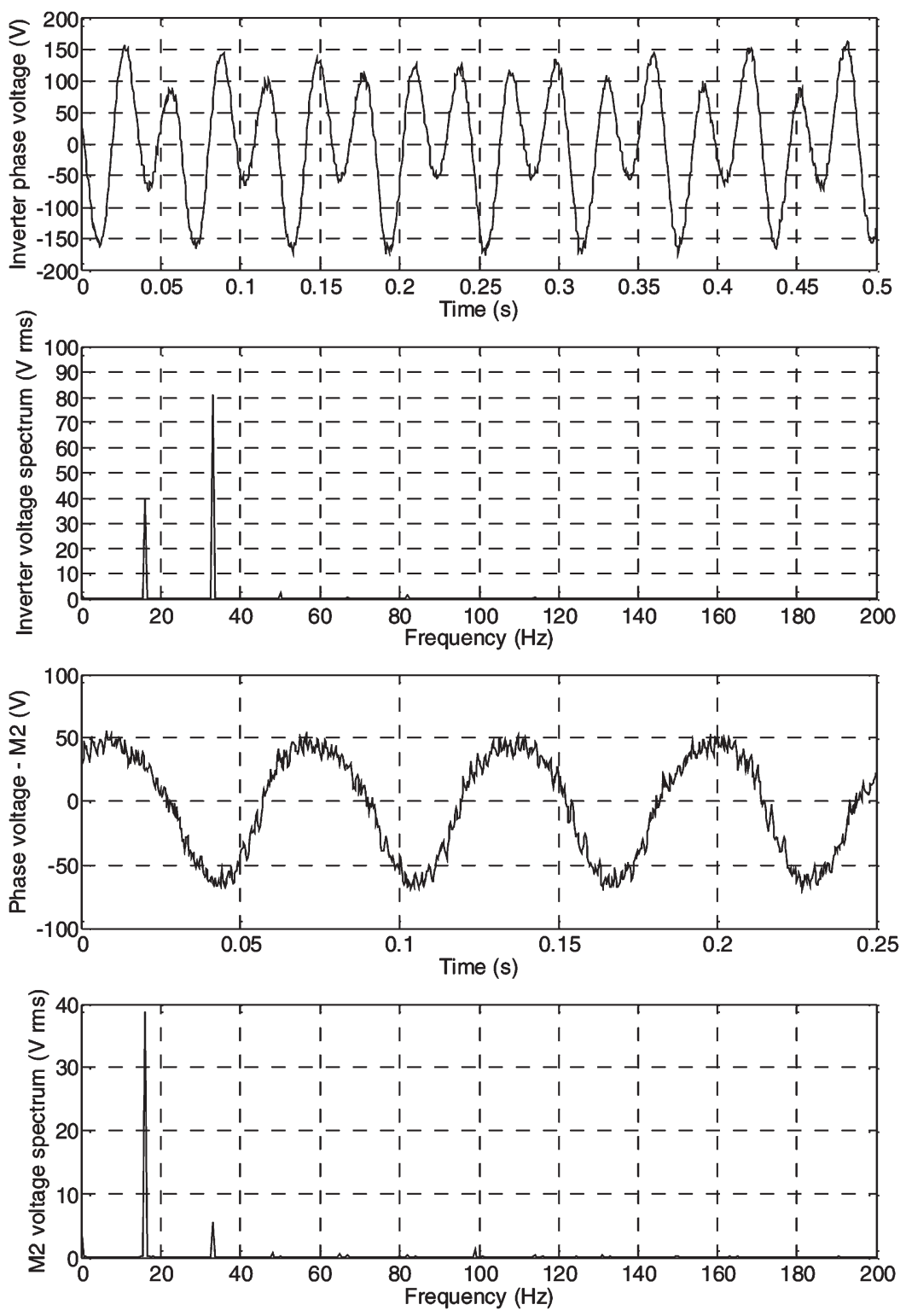

Fig. 13. Measured inverter and M2 phase voltages (waveforms and spectra) for the frequency pair $f_{1}=33 \mathrm{~Hz}$ and $f_{2}=16 \mathrm{~Hz}$.

Inverter phase currents under all operating conditions contain two components at two different frequencies, where the component at $f_{1}$ creates flux and torque in $\mathrm{M} 1$, while the component at $f_{2}$ yields flux and torque in $\mathrm{M} 2$. The waveform of the inverter current is similar to the inverter phase voltage. For this reason measured inverter current $i_{\mathrm{A}}$ is shown in Fig. 16 for one case only, operation with $f_{1}=20 \mathrm{~Hz}$ and $f_{2}=25 \mathrm{~Hz}$.

The presented experimental results fully verify the ability of the SVPWM scheme to generate the required voltage references in the two planes, regardless of the mutual relationship of the reference magnitudes.

\section{EXTENSION OF SVPWM TO Higher Phase Numbers}

The SVPWM methods presented in Section IV for the multifrequency output voltage generation of a five-phase VSI can be extended to higher phase numbers, starting from the corresponding sinusoidal SVPWM (i.e., [8] for a seven-phase VSI, [9] for a nine-phase VSI, etc.).

For example, if a seven-phase VSI is considered $(n=7)$, there are three independent voltage vector references, since there are $(n-1) / 2=3 d-q$ planes. The number of active space vectors selected, using the sinusoidal SVPWM principles [8], is $n-1=6$ in each of the three $d-q$ planes. Hence, the initial selection will involve $(n-1)^{2} / 2=18$ active space vectors. The switching period would further be subdivided into three (rather than two) subperiods, and one would have 18 expressions of the type shown in (9)-(12) (rather than 8). Subsequent modifications of the method 2, described in Section IV-C, would again produce a symmetrical switching pattern of the type illustrated in Fig. 7 (for seven rather than five inverter legs) and six active space vectors would ultimately be applied (where again some of them may be vector(s) that were not at all initially selected). The implementation principle, depicted in Fig. 11, remains the same, except that there would 

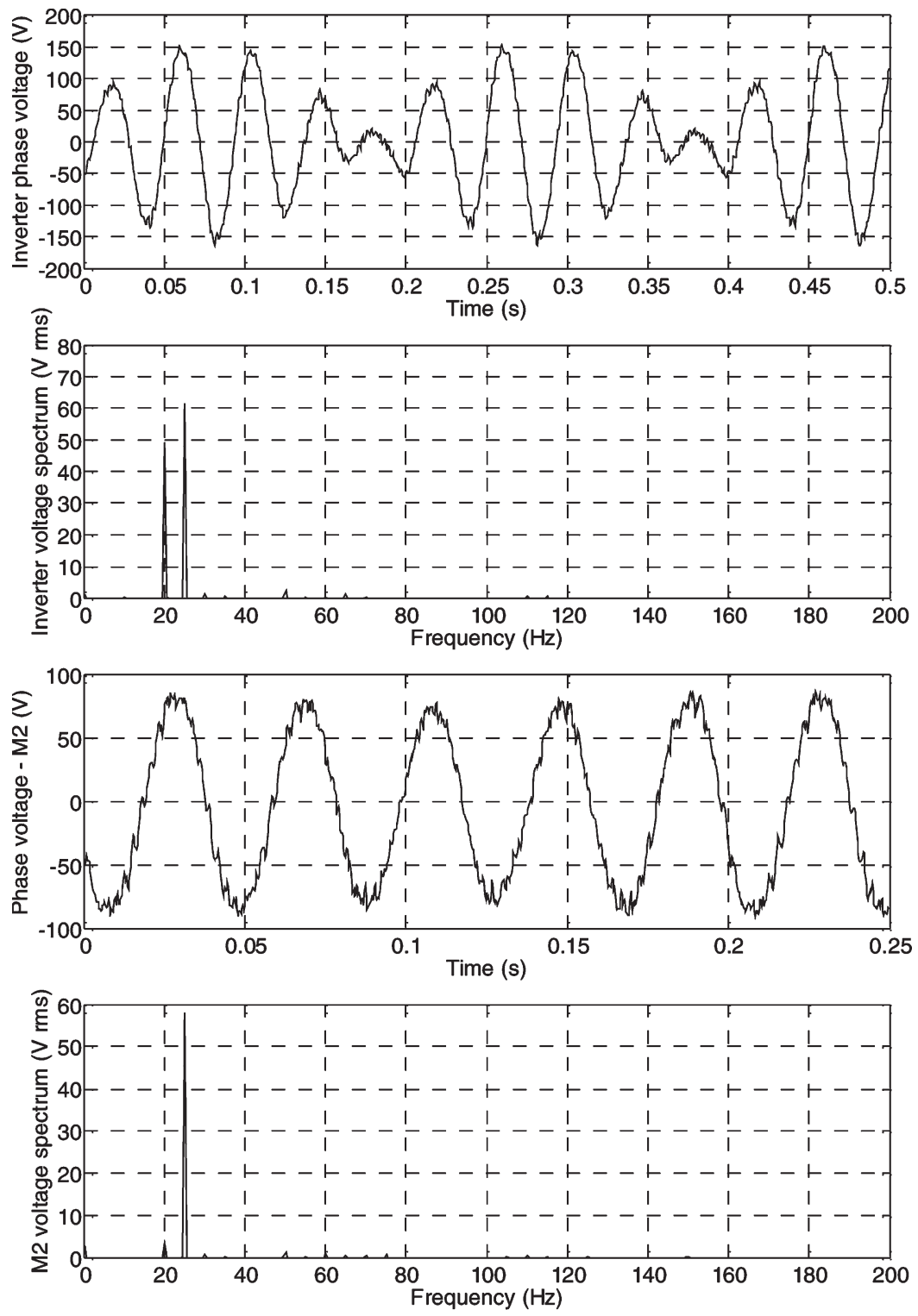

Fig. 14. Measured inverter and M2 phase voltages (waveforms and spectra) for the frequency pair $f_{1}=20 \mathrm{~Hz}$ and $f_{2}=25 \mathrm{~Hz}$.

be three voltage vector references (rather than two) at the input of, now, three (rather than two) space vector modulators on the left-hand side.

\section{CONCLUSION}

This paper has developed an SVPWM method for multiphase VSIs that is capable of generating required voltage references in all 2-D subspaces without restricting a priori achievable reference magnitudes in planes other than $d_{1}-q_{1}$. This is achieved by selecting initially $(n-1)^{2} / 2$ active space vectors within a switching period, instead of the customary $(n-1)$ active vectors. More specifically, a set of $(n-1)$ active vectors are selected in each of the $(n-1) / 2$ planes, in a manner that follows previously established rules for single-frequency output voltage generation with multiphase VSIs. It is shown that, by a suitable manipulation of the initially selected $(n-1)^{2} / 2$ active space vectors, it is possible to arrive at the SVPWM scheme where only $(n-1)$ active vectors are eventually applied. However, the vectors that are finally utilized are not known in advance and some may even not be among the initially selected set of $(n-1)^{2} / 2$ vectors.

Detailed considerations are given in this paper for the case of the five-phase VSI. Since there are two planes, it is possible to generate two voltage vector references of different frequencies. It has been shown that the SVPWM principles developed in this paper can be extended to higher phase numbers in a straightforward manner.

The developed SVPWM scheme has been experimentally verified using a two-motor series-connected five-phase drive, where the two voltage space vector references are required to independently control the two machines. These two references appear in two $d-q$ planes and are mutually unrelated in terms of phase, frequency and magnitude. However, the method is equally applicable to the control of concentrated winding machines, where one deliberately wants to produce certain voltage harmonic(s), which are in a fixed relationship to the fundamental in terms of magnitude, frequency and phase, for the sake of 

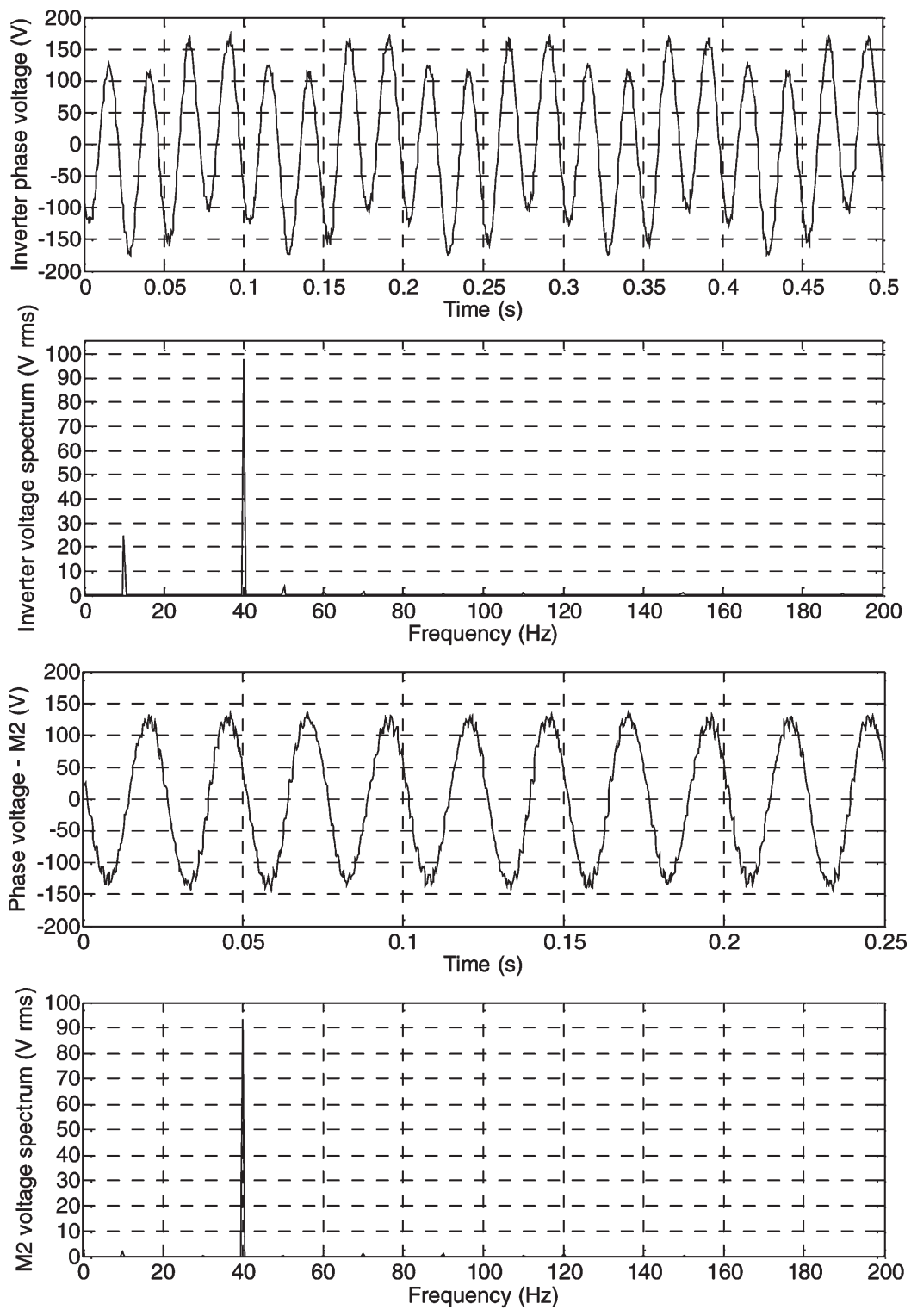

Fig. 15. Measured inverter and M2 phase voltages (waveforms and spectra) for the frequency pair $f_{1}=10 \mathrm{~Hz}$ and $f_{2}=40 \mathrm{~Hz}$.

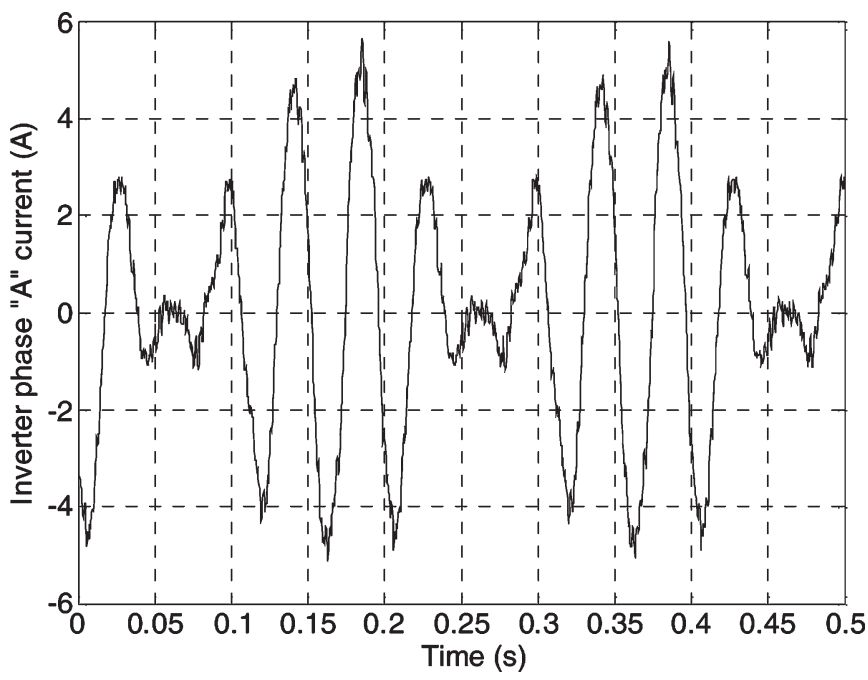

Fig. 16. Measured inverter current for operation with $f_{1}=20 \mathrm{~Hz}$, $f_{2}=25 \mathrm{~Hz}$. torque enhancement. Odd harmonics of the order lower than the phase number $n$ are then utilized (the third in a five-phase machine, the third and the fifth in a seven-phase machines, etc.). In such cases, in addition to the fundamental in the $d_{1}-q_{1}$ plane, there are again references in the other planes (in a five-phase machine the third harmonic gives a reference in the $d_{2}-q_{2}$ plane, etc.). The only thing that changes, compared to what has been considered in detail in this paper, is that the references in various planes are not independent any more. Everything else, including both theoretical and implementation aspects, remains the same. Since the implementation complexity of the existing SVPWM algorithms for higher harmonic injection in concentrated winding machines is rather high, while this algorithm is not computationally intensive, it is believed that the developed SVPWM scheme represents a good solution not only for multiphase multimotor drives with single VSI supply, but also for concentrated winding single-motor drives with higher harmonic injection. 


\section{REFERENCES}

[1] E. Levi, R. Bojoi, F. Profumo, H. A. Toliyat, and S. Williamson, "Multiphase induction motor drives-A technology status review," IET Elect. Power Appl., vol. 1, no. 4, pp. 489-516, Jul. 2007.

[2] Y. Zhao and T. A. Lipo, "Space vector PWM control of dual three-phase induction machine using vector space decomposition," IEEE Trans. Ind. Appl., vol. 31, no. 5, pp. 1100-1109, Sep./Oct. 1995.

[3] G. Grandi, G. Serra, and A. Tani, "General analysis of multiphase systems based on space vector approach," in Proc. Int. Conf. EPE-PEMC, Portoroz, Slovenia, 2006, pp. 834-840.

[4] J. W. Kelly, E. G. Strangas, and J. M. Miller, "Multiphase space vector pulse width modulation," IEEE Trans. Energy Convers., vol. 18, no. 2, pp. 259-264, Jun. 2003.

[5] A. Iqbal and E. Levi, "Space vector PWM techniques for sinusoidal output voltage generation with a five-phase voltage source inverter," Electr. Power Compon. Syst., vol. 34, no. 2, pp. 119-140, Feb. 2006.

[6] P. S. N. de Silva, J. E. Fletcher, and B. W. Williams, "Development of space vector modulation strategies for five-phase voltage source inverters," in Proc. IEE PEMD, Edinburgh, U.K., 2004, pp. 650-655.

[7] S. Xue and X. Wen, "Simulation analysis of two novel multiphase SVPWM strategies," in Proc. IEEE ICIT, Hong Kong, 2005, pp. 1401-1406.

[8] G. Grandi, G. Serra, and A. Tani, "Space vector modulation of a sevenphase voltage source inverter," in Proc. Int. SPEEDAM, Taormina, Italy, 2006, pp. S8-6-S8-13.

[9] G. Grandi, G. Serra, and A. Tani, "Space vector modulation of a ninephase voltage source inverter," in Proc. IEEE ISIE, Vigo, Spain, Jun. 4-7, 2007, pp. 431-436.

[10] R. O. C. Lyra and T. A. Lipo, "Torque density improvement in a six-phase induction motor with third harmonic current injection," IEEE Trans. Ind. Appl., vol. 38, no. 5, pp. 1351-1360, Sep./Oct. 2002.

[11] H. M. Ryu, J. H. Kim, and S. K. Sul, "Analysis of multiphase space vector pulse width modulation based on multiple d-q spaces concept," IEEE Trans. Power Electron., vol. 20, no. 6, pp. 1364-1371, Nov. 2005.

[12] D. Hadiouche, L. Baghli, and A. Rezzoug, "Space-vector PWM techniques for dual three-phase AC machine: Analysis, performance evaluation, and DSP implementation," IEEE Trans. Ind. Appl., vol. 42, no. 4, pp. 1112-1122, Jul./Aug. 2006

[13] O. Ojo, G. Dong, and Z. Wu, "Pulse-width modulation for five-phase converters based on device turn-on times," in Conf. Rec. IEEE IAS Annu. Meeting, Tampa, FL, 2006, pp. 627-634.

[14] E. Semail and C. Rombaut, "New method to calculate the conduction durations of the switches in a n-leg 2-level voltage source inverter," presented at the European Power Electronics and Applications Conf. (EPE), Graz, Austria, 2001, Paper 626, CD-ROM.

[15] X. Kestelyn, E. Semail, and J. P. Hautier, "Multi-phase system supplied by SVM VSI: A new fast algorithm to compute duty cycles," EPE J., vol. 14, no. 3, pp. 25-31, Aug. 2004.

[16] E. Levi, M. Jones, S. N. Vukosavic, and H. A. Toliyat, "A novel concept of a multiphase, multimotor vector controlled drive system supplied from a single voltage source inverter," IEEE Trans. Power Electron., vol. 19, no. 2, pp. 320-335, Mar. 2004.

[17] E. Levi, M. Jones, S. N. Vukosavic, A. Iqbal, and H. A. Toliyat, "Modeling, control, and experimental investigation of a five-phase seriesconnected two-motor drive with single inverter supply," IEEE Trans. Ind. Electron., vol. 54, no. 3, pp. 1504-1516, Jun. 2007.

[18] A. Iqbal, S. Vukosavic, E. Levi, M. Jones, and H. A. Toliyat, "Dynamics of a series-connected two-motor five-phase drive system with a singleinverter supply," in Conf. Rec. IEEE IAS Annu. Meeting, Hong Kong, 2005, pp. 1081-1088.

[19] K. K. Mohapatra, R. S. Kanchan, M. R. Baiju, P. N. Tekwani, and K. Gopakumar, "Independent field-oriented control of two split-phase induction motors from a single six-phase inverter," IEEE Trans. Ind. Electron., vol. 52, no. 5, pp. 1372-1382, Oct. 2005.

[20] E. Levi, S. N. Vukosavic, and M. Jones, "Vector control schemes for series-connected six-phase two-motor drive systems," Proc. Inst. Electr. Eng.-Electr. Power Appl., vol. 152, no. 2, pp. 226-238, Mar. 2005.

[21] A. Iqbal, E. Levi, M. Jones, and S. N. Vukosavic, "A PWM scheme for a five-phase VSI supplying a five-phase two-motor drive," in Proc. IEEE IECON, Paris, France, 2006, pp. 2575-2580.

[22] A. Iqbal and E. Levi, "Space vector PWM for a five-phase VSI supplying two five-phase series-connected machines," in Proc. Int. Conf. EPEPEMC, Portoroz, Slovenia, 2006, pp. 222-227.

[23] E. Levi, M. Jones, S. N. Vukosavic, and H. A. Toliyat, "Steady state modelling of series-connected five-phase and six-phase two-motor drives," in Conf. Rec. IEEE IAS Annu. Meeting, Tampa, FL, 2006, pp. 415-422.
[24] J. Figueroa, J. Cros, and P. Viarouge, "Generalized transformations for polyphase phase-modulation motors," IEEE Trans. Energy Convers., vol. 21, no. 2, pp. 332-341, Jun. 2006.

[25] X. Kestelyn, E. Semail, and J. P. Hautier, "Vectorial multi-machine modeling for a five-phase machine," presented at the Int. Conf. Electrical Machines (ICEM), Bruges, Belgium, 2002, Paper 394, CD-ROM.

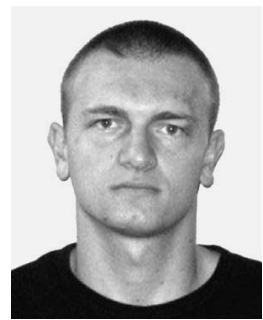

Drazen Dujic (S'03) received the B.Eng. and M.Sc degrees from the University of Novi Sad, Novi Sad, Serbia, in 2002 and 2005, respectively. Since 2006, he has been working toward the Ph.D. degree at Liverpool John Moores University, Liverpool, U.K.

He was with the Department of Electrical Engineering, University of Novi Sad, from 2002 to 2006 as a Research Assistant. His main research interest is in the area of high-performance electric motor drives.

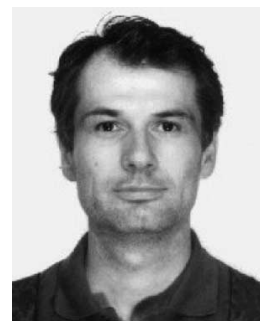

Gabriele Grandi (M'00) received the M.Sc. (cum laude) and Ph.D. degrees in electrical engineering from the Faculty of Engineering, University of Bologna, Bologna, Italy, in 1990 and 1994, respectively.

He became a Research Associate with the Department of Electrical Engineering, University of Bologna, in 1995. Since 2005, he has been an Associate Professor in the same department. His main research interests are focused on power electronic circuits and power electronic converters for renew-

able energy sources.

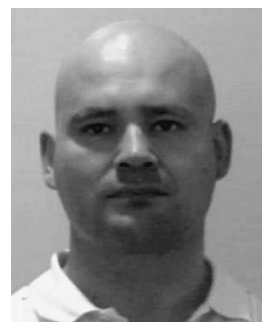

Martin Jones (M'07) received the B.Eng. (first class honors) and Ph.D. degrees from Liverpool John Moores University, Liverpool, U.K., in 2001 and 2005, respectively.

$\mathrm{He}$ was a Research Student with Liverpool John Moores University from September 2001 until Spring 2005, where he is currently a Postdoctoral Research Associate.

Dr. Jones was a recipient of the IEE Robinson Research Scholarship for his Ph.D. studies.

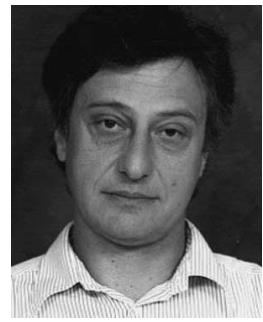

Emil Levi (S'89-M'92-SM'99) received the Dipl.Ing. degree from the University of Novi Sad, Yugoslavia, in 1982, and the M.Sc. and Ph.D. degrees from the University of Belgrade, Yugoslavia, in 1986 and 1990 , respectively.

From 1982 to 1992, he was with the Department of Electrical Engineering, University of Novi Sad. He joined Liverpool John Moores University, Liverpool, U.K., in May 1992, where, since September 2000, he has been a Professor of electric machines and drives.

Dr. Levi serves as an Editor of the IEEE TRANSACTIONS ON ENERGy CONVERSION, an Associate Editor of the IEEE TRANSACTIONS ON INDUSTRIAL ELECTRONICS, and as a member of the Editorial Board of the IET Electric Power Applications. 